\title{
Historia, precursores y actualidad de los estudios sobre el Sudeste de Asia en Argentina
}

Artículo científico. Material original autorizado para su primera publicación en el Journal de Ciencias Sociales, Revista Académica de la Facultad de Ciencias Sociales de la Universidad de Palermo. Buenos Aires. Argentina.

Dra. M. Florencia Rubiolo (CONICET/USiglo21) ${ }^{1}$ y Lic. Ezequiel Ramoneda (UNLP) ${ }^{2}$

Recibido: 14-9-2015

Aceptado: 7-10-2015

\section{Resumen:}

Los estudios sobre el Sudeste de Asia en Argentina se encuentran en un incipiente estadio de desarrollo. Aunque se han fortalecido los vínculos bilaterales con la región, de la mano de un floreciente intercambio comercial que ha comenzado a repercutir en las acciones de política externa tanto de Argentina como de los países contraparte, el tratamiento académico sobre cuestiones relacionadas con el Sudeste de Asia aún es mínimo. No obstante, la última década ha sido testigo de un mayor número de iniciativas académicas que, aunque en su mayor parte aisladas entre sí, representan un nuevo ímpetu en los estudios sobre la región asiática en nuestro país. Entendemos que este impulso tiene una estrecha vinculación con el dinamismo comercial y político que se ha evidenciado en las relaciones entre Argentina y el SEA en los últimos años.

Nuestro objetivo en este trabajo es realizar un diagnóstico del estado de los estudios sobre el Sudeste de Asia en la Argentina en la actualidad. Estimamos pertinente para ello realizar una breve introducción a la historia de las relaciones entre Argentina y los países del SEA para luego abordar los desarrollos y las iniciativas que se han implementado desde 2000 en adelante. De esta manera, se espera resaltar las principales motivaciones que impulsaron los primeros trabajos y subrayar los desafíos a superar en vistas a una profundización del conocimiento de las problemáticas intrínsecas de la región, de modo que contribuya a la toma de decisiones políticas y empresariales argentinas.

Palabras clave: Sudeste de Asia, Argentina, estudios académicos, política exterior

\footnotetext{
${ }^{1}$ María Florencia Rubiolo es Doctora en Relaciones Internacionales por la Universidad Nacional de Rosario. Es investigadora asistente de CONICET, Directora de proyecto de Investigación en la Universidad Siglo 21 y docente de la Facultad de Ciencia Política y Relaciones Internacionales de la UCC. Su actual línea de investigación es la política exterior y vinculaciones comerciales de Argentina con el Sudeste de Asia, China e India. Mail: frubiolo@gmail.com

${ }^{2}$ Ezequiel Ramoneda es Licenciado en Estudios Orientales (USAL). Coordinador del Centro de Estudios del Sudeste Asiático del Departamento de Asia y el Pacifico del Instituto de Relaciones Internacionales de la Universidad Nacional de la Plata.
} 


\section{Abstract:}

Studies on Southeast Asia are in a very early development stage in Argentina. Although bilateral bonds have been fostered with that region, together with flourishing commercial exchanges, with an increasing impact in foreign policies of Argentina as well in its counterparts, academic treatment on issues related to Southeast Asia is yet minimum. In spite of that, the last decade has witnessed a higher amount of academic initiatives that, even though mostly in an isolated way, represent a new momentum in studies on the Asian region in our country, We understand that momentum has a tight bond with the commercial and political dynamism demonstrated in Argentina-Southeast Asian relations in the last years.

Our objective in this paper is to perform a diagnostic on the state-of-the-art of studies on Southeast Asia right now. We consider it relevant to briefly introduce the history of relationships between Argentina and the countries in the Asian region, in order to address later the subsequent developments and initiatives that have been implemented since 2000 on. This way we expect to highlight the main causes that inspired the first works, and emphasize the challenges to be overcome in view of a deepening of the knowledge of the region's essential problems that contribute to Argentine political and business decision making.

Keywords: Southeast Asia, Argentina, academic research, foreign policy.

\section{Introducción}

Los estudios sobre el Sudeste de Asia en Argentina se encuentran en un incipiente estadio de desarrollo. Aunque se han fortalecido los vínculos bilaterales con la región, de la mano de un floreciente intercambio comercial que ha comenzado a repercutir en las acciones de política externa tanto de Argentina como de los países contraparte, el tratamiento académico sobre cuestiones relacionadas con el Sudeste de Asia aún es mínimo. Con algunas excepciones, la región ha pasado inadvertida en los análisis de política exterior argentina y en los de relaciones Sur-Sur, entre otros.

No obstante, observamos que en los últimos años se evidencia un mayor interés desde la docencia y la investigación, en cuestiones que engloban al Sudeste de Asia. En la mayor parte de las oportunidades, el tratamiento de la región se produce en el marco de estudios sobre el Este de Asia, o Asia Oriental, en los cuales los trabajos sobre China concentran la principal atención. Se refleja así la dificultad para definir un espacio autónomo para los estudios del Sudeste de Asia, que suelen encontrarse subsumidos en áreas de interés geográficamente más amplias.

Nuestro objetivo en este trabajo es realizar un diagnóstico del estado de los estudios sobre el Sudeste de Asia en la Argentina en la actualidad. Estimamos pertinente para ello realizar una breve introducción a la 
historia de las relaciones entre Argentina y los países del SEA para luego abordar los desarrollos y las iniciativas que se han implementado desde 2000 en adelante. De esta manera se espera resaltar las principales motivaciones que impulsaron los primeros trabajos, y subrayar los desafíos a superar en vistas a una profundización del conocimiento de las problemáticas intrínsecas de la región que contribuya a la toma de decisiones políticas y empresariales argentinas.

\section{Historia de las Relaciones entre Argentina y el Sudeste Asiático}

Históricamente, la República Argentina ha sido uno de los primeros países en América Latina en establecer relaciones diplomáticas con los nuevos estados del Sudeste Asiático. Salvo el Reino de Tailandia, que logró mantenerse independiente, varios de los pueblos del Sudeste Asiático llevaron adelante procesos exitosos de resistencia y autodeterminación contra las potencias coloniales occidentales, que intentaron retomar su dominio sobre estos tras el fin de la Segunda Guerra Mundial (Bianco, 1995; Panikkar, 1966). De esta manera, la República Argentina estableció formalmente relaciones diplomáticas con la República de Filipinas el 27 de agosto de 1948, con el Reino de Tailandia el 2 de febrero de 1955, con la República de Indonesia el 30 de julio de 1956, con la República de Vietnam (Vietnam del Sur) el 19 de septiembre de 1959, luego reformuladas con la República Democrática de Vietnam (Vietnam del Norte) el 15 de octubre de 1973 (tras el fin de la Guerra de Vietnam se renombró República Socialista de Vietnam), con el Reino de Camboya el 28 de enero de 1960, con Malasia el 7 de junio de 1967, con la República de Singapur el 12 de septiembre de 1974, con la República Democrática Popular Lao el 13 de noviembre de 1975, y finalmente con la República Socialista de la Unión de Myanmar el 10 de enero de 1979 (MREC, 2015).

Ahora bien, lo anterior no hace referencia a los primeros contactos históricos entre el pueblo Argentino y los pueblos de aquella región asiática. Con el establecimiento del Imperio Español en el continente americano desde mediados del siglo XVI, se articularon una serie de rutas comerciales que vincularon la Capitanía General de Filipinas, archipiélago asiático que también estuvo bajo el dominio colonial español, con el Virreinato de Nueva España (México) en América del Norte y el Virreinato del Perú en América del Sur. Con el tiempo estas rutas llegarían hasta la región del Río de la Plata, situación que se vería consolidada con el establecimiento del Virreinato del Río de la Plata ya para fines del siglo XVIII. De aquí que el archipiélago filipino fue el punto de contacto de los pueblos latinoamericanos con los pueblos de aquella región asiática. Fue por medio de estas rutas que algunos "Indios de la China" Ilegaron a Sudamérica en general, y a los territorios rioplatenses en particular, quienes en algunos casos terminaron radicándose (Braccio, 2009). Existían sedes de la Compañía Real de Filipinas en distintas ciudades del Imperio Español en 
América, en particular una en la ciudad de Buenos Aires cuando era capital del Virreinato del Río de la Plata, la cual contaba con un depósito de esclavos en los terrenos del actual Parque Lezama (Crespi, 2001; Crespi, 2010; Mariluz Urquijo, 1984; O’ Donnell, 1997).

Años después, con el desarrollo de la independencia de los pueblos de la región latinoamericana, se inició el proceso de desarticulación del monopolio español de estas rutas comerciales terrestres y marítimas. Las Provincias Unidas del Río de la Plata, antecesoras de la República Argentina, se declararon independientes de España el 9 de julio de 1816, y la armada del nuevo estado rioplatense tuvo su participación en el proceso previamente mencionado. Poco después de la declaración de independencia en el año 1816, el Almirante Guillermo Brown siguió adelante una política de guerra marítima basada en el corso contra las fuerzas marítimas españolas tanto en el océano Atlántico como también en el océano Pacífico. En el marco de ésta es importante destacar la gesta del Capitán Hipólito Bouchard quien, al mando de la Fragata "La Argentina”, zarpó en 1817 hacia las aguas del Sudeste Asiático para luchar contra los españoles en Filipinas, logrando sitiar el puerto de Manila (Caillet-Bois, 1930; Cichero, 1999; Medina, 1928; O’ Donnell, 2004; O’Donnell, 2006; Polverini, 1968; Quartaruolo, 1967). No sería la única vez en la que Argentina tuviera presencia en aquellas aguas, ya que desde fines del siglo XIX la fragata Presidente Sarmiento navegaría las mismas en varias ocasiones.

Ya entrado el siglo XX, las relaciones diplomáticas entre Argentina y los países del Sudeste Asiático desde el fin de la Segunda Guerra Mundial a mediados de la década del cuarenta hasta principios de la década del noventa fueron enmarcadas en el orden mundial bipolar de la Guerra Fría. Este periodo estuvo caracterizado por una puja entre esquemas autonomistas y esquemas de alineamiento con Estados Unidos. De acuerdo con Simonoff (2010a), los primeros esquemas fueron llevados en su mayoría por administraciones democráticas -hasta Menem-, y los segundos, por gobiernos de facto. Sin embargo, estas orientaciones no fueron estáticas ni libres de vaivenes, generando una falta de continuidad entre gobiernos y también dentro de un mismo gobierno. En el caso de las relaciones con el Sudeste de Asia, los vínculos fueron esporádicos. Esto respondió a la escasa relevancia que la región tenía, tanto en la dimensión política, como en la económica, para nuestro país.

No obstante, el estado Argentino ha establecido relaciones diplomáticas con los estados de la región del Sudeste Asiático que, comparativamente, son más profundas y complejas que las de otros países latinoamericanos.

En primer lugar, cuando Argentina participó por primera vez como miembro no permanente del Consejo de Seguridad de las Naciones Unidas entre los años 1948 y 1949, al tratarse, entre otros temas de la agenda, la lucha por la independencia del pueblo indonesio contra el dominio colonial holandés. No fue así para la 
lucha por la independencia del pueblo vietnamita contra el dominio colonial francés por oposición de Francia. Ambas potencias coloniales invocaron la jurisdicción doméstica para la consideración contraria de la lucha por la independencia por tener lugar dentro de lo que consideraban parte de su territorio (colonial). Estos eventos fueron contemporáneos a la primera y la segunda presidencias de Juan Domingo Perón, entre los años 1946-1955. Los postulados teóricos de la orientación de la política exterior durante estos gobiernos de Perón fueron enmarcados en la Tercera Posición, alternativa al capitalismo y el comunismo. Ahora bien, aunque teóricamente aparentaba tener implicancias en lo que sería el desarrollo posterior del Tercer Mundo, no tuvo incidencia real en los acontecimientos que estaban teniendo lugar en Asia para ese entonces. En la misma pesaron la tradición legalista y de no intervención en los asuntos de jurisdicción interna de los estados, su orientación geográfica hacia América Latina y Europa, a la vez que los intereses económicos con respecto al acceso comercial a los mercados europeos y el estímulo financiero estadounidense (Oviedo, 2001; Panikkar, 1966).

También en esta línea se inscribe la presidencia de Arturo Frondizi (1958-1962) que, en pos de la consecución de una política de orientación desarrollista, privilegió en política exterior la apertura de nuevos mercados, aunque en aquellos países pro-occidentales. Una de las acciones que se inscribió en estos lineamientos fue la visita a Tailandia, Vietnam (del Sur) y Filipinas, en una gira presidencial que se realizó en 1961. Este viaje convirtió a Frondizi en el primer Presidente argentino que llevó a cabo una visita oficial al Sudeste de Asia. La comitiva, que comprendió al Ministro de Relaciones Exteriores Miguel Ángel Cárcano, altos funcionarios civiles y militares, periodistas y veintiocho representantes de diferentes sectores productivos del país, visitó Canadá, Grecia, India, y Japón, además de los países del Sudeste Asiático mencionados. ${ }^{3}$ De acuerdo con fuentes de la época, el principal interés perseguido en esta iniciativa era la apertura de nuevos mercados para los productos argentinos (El Tiempo, 1961). La gira, que comenzó el 27 de noviembre y finalizó el 24 de diciembre, buscó la diversificación de mercados para los productos argentinos en Asia, un continente inexplorado hasta ese momento para la Argentina. La magnitud del mercado oriental y su potencial de crecimiento justificaban, a los ojos del entonces primer mandatario, una visita sin precedentes a la región con el objetivo de estimular los nimios intercambios que en ese momento se mantenían con Oriente ${ }^{4}$. Incluso durante su estadía en Tailandia, dirigió palabras en la sede de la Organización del Tratado del Sudeste Asiático en la ciudad de Bangkok (Oviedo, 2001; Pelosi y Machinandiarena de Devoto, 2012).

\footnotetext{
${ }^{3}$ El viaje culminó con una breve parada en Honolulu y luego en el Estado de Florida donde mantuvo una reunión con el entonces Presidente de Estados Unidos, Kennedy (US Department of State, 2012).

${ }^{4}$ Asimismo, "El presidente entendía que este viaje cerraba el periplo iniciado con los realizados a Europa y países de América, y era el complemento indispensable de la acción que el gobierno estaba ejerciendo en el exterior" (Devoto, Pelosi, 2010, p. 150).
} 
Pocos años después, el Canciller Miguel Ángel Zavala Ortiz realizó también una visita a Tailandia (Embajada Argentina en Bangkok, 2005) y una escala en Vietnam del Sur en 1966, luego de atender a la reunión de la Comisión Económica para Asia y Lejano Oriente (ECAFE, por sus siglas en inglés, en la actualidad, ESCAP) en Japón. Simonoff señala que la visita a Vietnam del Sur puso en evidencia las diferencias entre el Canciller Zavala Ortiz y el Presidente Illia, dado que la presencia del primero en ese país en pleno desarrollo del conflicto con Vietnam del Norte daba a entender el apoyo a los intereses de Estados Unidos en la contienda, mientras Illia criticaba el conflicto (Simonoff, 2010).

Argentina volvió a participar en el Consejo de Seguridad entre los años 1966 y 1967 como también los años 1972 y 1973, años contemporáneos a la Guerra de Vietnam. Nuevamente fue la oposición de los Estados Unidos lo que impidió que la lucha del pueblo vietnamita fuese incorporada para su tratamiento en la agenda del Consejo de Seguridad. Con relación a los eventos que estaban teniendo lugar en ese país, el gobierno argentino envió alimentos y medicamentos a Vietnam del Sur en el año 1966. Ahora bien, fue la solidaridad brindada al pueblo vietnamita por parte del pueblo argentino lo que distinguió la relación entre Argentina y Vietnam en estos años. En 1966 se creó el Movimiento Argentino de Ayuda al Vietnam (MAViet), que en 1968 se recicló en el de Solidaridad Argentina con Vietnam. Este movimiento social llevó adelante, hasta su disolución en el año 1978, una serie de iniciativas para pujar por el fin de la intervención de los Estados Unidos en Vietnam, como la realización de un petitorio con poco más de 67.000 firmas presentado en la Embajada de los Estados Unidos en Argentina para ser entregado al presidente Richard Nixon exigiendo el fin de la guerra, como también ayudar a paliar el sufrimiento del pueblo vietnamita, como la recolección de fondos entre los años 1968 y 1971 por valor de más de 30 millones de pesos argentinos para la compra de medicamentos, especialmente quinina, para ser entregados en Vietnam del Norte, por medio de la Cruz Roja Internacional, para paliar los efectos del agente naranja, y la publicación de gacetillas y folletos sobre los acontecimientos de la guerra en Argentina con una mirada sin el sesgo de los medios occidentales (Dirección Nacional de Solidaridad Argentina con Vietnam, 1966; Dirección Nacional de Solidaridad Argentina con Vietnam, 1968; Dirección Nacional de Solidaridad Argentina con Vietnam, 1969; Dirección Nacional de Solidaridad Argentina con Vietnam, 1970; Dirección Nacional de Solidaridad Argentina con Vietnam, 1971). Vale destacar la participación dentro del Movimiento de Solidaridad Argentina con Vietnam del Partido Comunista Argentino. El Partido Comunista Argentino mantiene largas relaciones con el Partido Comunista de Vietnam. En el año 1924 Miguel Contreras, uno de los fundadores del Partido, entabló amistad con Ho Chi Minh durante su participación en el V Congreso de la Internacional Socialista celebrado en Moscú. Finalmente en octubre de 1973, durante la tercera presidencia de Juan Domingo Perón (19731974) se descontinuaron los contactos diplomáticos con Vietnam del Sur, y se establecieron relaciones 
diplomáticas con Vietnam del Norte. Para este entonces se puede apreciar una mayor sintonía entre la Tercera Posición como orientación de la política exterior de Perón y la realidad del Tercer Mundo. El reconocimiento de Vietnam del Norte fue luego de la firma de los acuerdos de paz de París en enero de 1973, pero antes del desenlace final de la Guerra de Vietnam en abril de 1975, en un nuevo ejemplo del respeto de la tradición legalista argentina, pero en una nueva expresión de autonomía, siendo este una expresión del acercamiento argentino al mundo socialista. En esta línea, en ese mismo año Argentina formaliza su participación dentro del Movimiento de Países no Alineados, en el cual se había incorporado como miembro observador en el año 1964, pero ahora se convierte en miembro pleno (Oviedo, 2001; Saavedra, 2004).

Durante el gobierno de facto de Jorge Rafael Videla (1976-1981) el gobierno militar orientó la política exterior del país nuevamente hacia el ámbito occidental. Pero así al buscar limpiar su imagen internacional en derechos humanos, se hizo eco de una manera pragmática de la crisis humanitaria producto del fin de la situación bélica en los nuevos países de la antigua Indochina Francesa y el establecimiento en los mismos de gobiernos comunistas. Por medio de un acuerdo con el Alto Comisionado de las Naciones Unidas para los Refugiados, entre septiembre de 1979 y marzo de 1980, el gobierno argentino aceptó recibir 1243 refugiados indochinos, siendo el único país latinoamericano en hacerlo. Con el tiempo esta cantidad menguaría debido a la inoperancia de su selección como también la falta de preparación para su recepción en el país, pero un núcleo duro decidió permanecer y establecerse (Dirección Nacional de Población, 2012). Los vínculos entre la Argentina y el SEA se mantuvieron en un escaso nivel de desarrollo y una genuina irrelevancia para ambas partes hasta avanzada la década del ochenta. La ausencia de visitas oficiales desde el SEA hacia nuestro país puso de manifiesto el bajo nivel de prioridad que la Argentina tenía en la agenda de los países asiáticos. Recién en 1981 se recibió la primera visita oficial de la región con el arribo del Ministro de Relaciones Exteriores de Tailandia, General Sit Sawetsila (Embajada Argentina en Bangkok, 2005). Con ocasión del encuentro se firmó un Convenio para la Cooperación Científica y Técnica. Desde entonces y hasta la década del noventa no volvieron a recibirse visitas oficiales desde el Sudeste de Asia.

De parte de la Argentina, el Presidente Alfonsín realizó una visita a Filipinas donde fue recibido por la Presidente Corazón Aquino en julio de 1986. Con este país también se firmó un convenio comercial en 1984 previsto en el tratado de Amistad y Relaciones Culturales de 1960 (Oviedo, 2000). El acuerdo, tendiente a fortalecer las relaciones comerciales entre ambas partes, hacía especial hincapié en la necesidad de promover el intercambio de productos manufacturados y semi-manufacturados con la intención de diversificar el comercio bilateral (MREC, 1984). 
Entrada la década del noventa, marcada por el fin de la Guerra Fría, surge un nuevo contexto del orden mundial. La redefinición de las directrices de política exterior y particularmente el perfil más internacionalista de la nueva administración, tuvieron cierto impacto en las vinculaciones con el Sudeste de Asia. En este sentido, el presidente Carlos Menem realizó dos giras presidenciales por el Sudeste de Asia, en 1996 y en 1997. La primera de ellas tuvo como países de destino Malasia e Indonesia, y la segunda se dirigió a Tailandia y Singapur. Las comitivas en ambos viajes fueron integradas por un nutrido grupo de empresarios, evidenciando el fuerte contenido comercial de las acciones de política exterior hacia el SEA.

Debe destacarse que durante este período se firmaron numerosos acuerdos de cooperación económica y comercial (con Filipinas el 29 de junio de 1984, con Indonesia el 9 de octubre de 1990, con Malasia el 1 de julio de 1991, con Vietnam el 3 de junio de 1996, y con Tailandia el 19 de febrero de 1997) y de promoción y protección recíproca de inversiones (firmado con Malasia el 6 de septiembre de 1994, con Indonesia el 7 de noviembre de 1995, con Vietnam el 3 de junio de 1996, con Filipinas el 20 de septiembre de 1999, y con Tailandia el 18 de Febrero de 2000) (MREC, 2015).

Ahora bien, las relaciones entre Argentina y la región sufrieron los efectos de la crisis asiática de los años 1997-1998, cuyo epicentro y mayores repercusiones tuvieron lugar en las economías del Sudeste Asiático. Poco tiempo después, la crisis Argentina llevó a un retraimiento del país de la escena internacional y a una reducción de las acciones de política exterior hacia el SEA. Sería con la presidencia de Néstor Kirchner (20032007) que comienza a haber una reactivación de las vinculaciones comerciales, la cual se vio fortalecida durante las dos presidencias de Cristina Fernández de Kirchner (2007-2015). Esta última mandataria visitó Indonesia y Vietnam en el mes de enero del año 2013. Durante las presidencias del matrimonio Kirchner entrarían en vigor los acuerdos de cooperación económica y comercial, y de promoción y protección recíproca de inversiones previamente mencionados, como también se firmarían la mayoría de los memorandos de entendimiento sobre consultas bilaterales/políticas (con Vietnam el 3 de junio de 1996, con Filipinas el 17 de febrero de 2005, con Indonesia el 12 de agosto de 2005, Tailandia el 19 de septiembre de 2006, con Singapur el 26 de agosto de 2011, con Laos el 27 de agosto de 2014) y de cooperación técnica (con Tailandia el 20 de octubre de 1981, con Malasia el 1 de Julio de 1991, con Indonesia el 24 de agosto de 2011, con Filipinas el 24 de agosto de 2011, con Vietnam el 17 de febrero de 1997 y el 26 de agosto de 2011, con Laos el 26 de agosto de 2011, con Camboya el 24 de septiembre de 2012) (MREC, 2015).

Entrado el nuevo siglo, el principal ámbito multilateral de vinculación de Argentina con los países del Sudeste Asiático es el Foro de Cooperación de América Latina y Asia del Este (FOCALAE). En este marco, en los últimos años se han implementado proyectos de cooperación técnica por medio del Fondo Argentino de Cooperación Sur Sur y Triangular (FO.AR) con varios países del Sudeste Asiático, principalmente en el área 
de agricultura y ganadería, pero donde también destaca la transferencia de conocimientos del Equipo Argentino de Antropología Forense para la identificación de víctimas de la Guerra de Vietnam, entre otros proyectos.

\section{Historia de los Estudios del Sudeste Asiático en Argentina}

Luego de la breve revisión de las relaciones bilaterales entre Argentina y el SEA, no realizaremos una aproximación al estado del arte de los estudios sobre el Sudeste de Asia en nuestro país. Para facilitar tal propuesta, y brindar una noción de continuidad y evitar una simple enumeración, se considerará la pertenencia institucional de los mismos.

Como se mencionó anteriormente, la cuestión de los orígenes de los estudios del Sudeste Asiático en Argentina se sumerge en la misma historia de los estudios de relaciones internacionales y asiáticos en el país. En lo que hace a los estudios en relaciones internacionales, la primera referencia de una carrera de grado en relaciones internacionales en una universidad nacional se remite a la década de 1920 con la licenciatura en diplomacia y servicio consular de la Universidad Nacional del Litoral. Para una universidad privada recién habría una en la década de 1960 con el ofrecimiento de cursos por parte de la Escuela de Diplomacia de la Universidad del Salvador. En 1997 la Universidad de Belgrano crea la Maestría en Relaciones Internacionales. En este ambiente se empezó con una oferta académica especializada de posgrado para carreras de grado en ciencias políticas. La primera publicación concerniente al área fue la Revista de Derecho Internacional y Ciencias Diplomáticas de la Facultad de la Universidad Nacional del Litoral, luego continuada por la Universidad Nacional de Rosario, iniciada en 1949 (Bulcourf, Cardozo y Campos Ríos, 2013).

Con la década del ochenta, además de un incremento en la oferta de formación académica en relaciones internacionales a nivel tanto de grado como de posgrado en el ámbito universitario, comienzan a surgir centros de investigación. En el ámbito de las universidades públicas, en 1988 se creó el Centro de Estudios en Relaciones Internacionales de Rosario (CERIR) de la Facultad de Ciencia Política y Relaciones Internacionales de la Universidad Nacional de Rosario, que cuenta con tres publicaciones (Estudios, Documentos de Trabajo y Docencia). En 1989 se creó el Instituto de Relaciones Internaciones (IRI) de la Facultad de Ciencias Jurídicas y Sociales de la Universidad Nacional de la Plata, que cuenta con dos publicaciones (Relaciones Internacionales, y Anuario en Relaciones Internacionales). Y en el ámbito de las universidades privadas se destaca el Instituto de Investigación en Ciencias Sociales (IDICSO) de la Facultad de Ciencias Sociales de la Universidad del Salvador (Bulcourf, Cardozo y Campos Ríos, 2013). 
Ahora bien, en lo que hace a los estudios asiáticos, las primeras referencias no vendrán tanto desde el área de las Relaciones Internacionales sino desde el área de la Historia. En la década de 1960 empiezan a surgir cátedras de Historia de Asia y África en carreras de grado en Historia en algunas universidades nacionales, como la Universidad Nacional del Comahue y la Universidad Nacional de Rosario. También, en el ámbito privado, en 1968 se establece la cátedra de Historia Moderna de Asia y África en la Universidad del Salvador. Paralelamente se crea el Centro de Estudios Orientales en la Facultad de Filosofía, el cual en 1967 se eleva a la categoría de Escuela dependiendo directamente del rectorado y se estableció la Licenciatura en Estudios Orientales (Escuela de Estudios Orientales, 2015).

Estas iniciativas se continúan en la década de 1970. En 1975 la Universidad de Belgrano creó el Instituto de Asia y África. Para esta época surge la colección Biblioteca de Asia y África de la editorial EUDEBA de la Universidad de Buenos Aires, en la cual se publicaron trabajos relacionados con los países del Asia. Ya iniciada la década de 1980 hay un incremento de la oferta académica. Se empezaron a dictar las cátedras de Historia de la Colonización y Descolonización, e Historia de Asia y África Contemporánea en la Facultad de Filosofía y Letras de la Universidad de Buenos Aires, la cátedra Historia de Asia y África de la Facultad de Humanidades y Ciencias de las Educación de la Universidad Nacional de la Plata, la cátedra Historia de Asia, África y Oceanía de la Universidad Nacional de Luján, las cátedras de Historia de Asia y África I y II en la Facultad de Humanidades y Arte de la Universidad Nacional de Rosario, las cátedras de Formación del Mundo Afro-Asiático y Problemática Contemporánea de Asia y África en la Universidad Nacional del Litoral, la cátedra de Historia Contemporánea de Asia y África en la Facultad de Filosofía y Humanidades de la Universidad Nacional de Córdoba, la Cátedra Mundo Actual Afro-Asiático de la Universidad Nacional del Comahue. Para esta época se incorpora la publicación de la revista Temas de Asia y África de la Sección Interdisciplinaria de Estudios de Asia y África de la Universidad de Buenos Aires.

A lo anterior debe sumarse la cátedra de Historia de la Colonización y Descolonización de Asia y África en la Universidad de Morón (Buffa, 2008). También se estableció en 1980 el Instituto Latinoamericano de Investigaciones Comparadas Oriente Occidente (ILICOO), desde el cual se publicó la revista Oriente Occidente (Escuela de Estudios Orientales, 2015). Como último hito, en 1987 se efectuó en la ciudad de Buenos Aires la V edición del Congreso Internacional de la Asociación Latinoamericana de Estudios de Asia y África (ALADDA), que coincidió con la primera edición ese mismo año del Congreso Nacional de ALADAA (Buffa, 2008).

Hacia la década de 1990 empieza a darse la intersección entre las áreas de estudios internacionales y de estudios asiáticos, en sintonía con el cambio en el orden mundial, y el mayor atractivo principalmente económico de los países de Asia. Se pueden destacar el CERIR de la UNR, el IRI de la UNLP, donde en sus 
publicaciones regulares empiezan a aparecer investigaciones relativas a Asia. A estos viene a sumarse el Grupo de Estudios del Este Asiático del Instituto de Investigaciones Gino Germani de la Facultad de Ciencias Sociales de la Universidad de Buenos Aires creado en 2001 (UBA, 2015) el cual estableció la cátedra de China, Corea y Japón: Una Mirada Histórica, Política, Económica y Cultural del este Asiático; el Centro de Estudios sobre Asia del Pacífico e India (CEAPI) creado en 2012, el cual estableció el posgrado Especialización en Economía y Negocios con Asia del Pacífico e India (Universidad Nacional de Tres de Febrero, 2015), y el Centro de Estudios Avanzados de la Universidad Nacional de Córdoba, la cual estableció la Especialización en Estudios de Asia Oriental (Buffa, 2008; Paz, 2005). En lo que respecta a las actividades académicas con apoyo estatal, en el año 1992 se creó la Comisión Nacional de Asia y el Pacífico (CONAPAC) por decreto del presidente Menem, que funcionó hasta 1999. Su objetivo era articular las iniciativas de actores del ámbito académico, empresarial y estatal para establecer una política consistente y dinámica con respecto a Asia (Paz, 2005).

En el año 1999 se organizó una misión tripartita entre funcionarios del gobierno, empresarios y académicos, quienes visitaron algunos países de la región (Di Masi, 1999). Es para esa época que se firmaron los primeros memorandos de entendimientos entre universidades argentinas y universidades de algunos de los países del Sudeste Asiático, no obstante la crisis argentina truncó durante algún tiempo la implementación de los mismos. Se firmaron acuerdos entre la Universidad Nacional de la Plata con la Universidad de Chulalongkorn de Tailandia, la Universidad de Malaya de Malasia, la Universidad de Ciencia Sociales y Humanidades Universidad Nacional de Vietnam, la Universidad Comercial de Hanoi, y la Universidad de Comercio Exterior de Vietnam. También la Universidad del Salvador firmó acuerdos con la Universidad de Asunción de Tailandia y la Universidad del Ateneo de Manila de Filipinas.

Entrado el siglo XXI, observamos un florecimiento de las iniciativas académicas vinculadas al Este de Asia y un surgimiento de institutos y grupos de investigación, así como un incremento de la producción académica, vinculada directamente al Sudeste de Asia, diferenciando a la región del contexto más amplio del Este asiático. En el próximo aportado nos referimos específicamente a estas iniciativas de reciente surgimiento.

\section{El Sudeste de Asia en la academia argentina actual}

Durante los últimos años, ha habido una importante producción académica de ensayos y publicación de libros relacionados con los estudios del Sudeste Asiático. También es importante mencionar la creación del Instituto de Cultura Argentino Vietnamita en 1997, del Sitio de los Becarios del Programa de Cooperación Técnica de Malasia que reúne a los argentinos que fueron becados para formarse en Malasia en 2010, como 
también de Forindar - Instituto para el Fomento de las Relaciones Indonesio Argentinas en 2012. En el año 2013 se implementó un proyecto de vinculación entre universidades argentinas y tailandesas por medio de la asistencia de la Secretaria de Políticas Universitarias del Ministerio de Educación.

Teniendo presente lo anterior y tomando como eje la región del Sudeste Asiático, se propone plantear a modo de sugerencia e introducción al tema en cuestión, algunas iniciativas precursoras, aunque indirectas, de los estudios de la región del Sudeste Asiático.

Terminada la Guerra fría y comenzado el giro económico hacia Asia, se inician propiamente los estudios sobre el Sudeste Asiático, aunque de manera incipiente. Con el crecimiento del comercio hacia la región, se ha generado cierta motivación académica por estudiar los países de Asia Oriental, incluidos los del Sudeste Asiático.

En la primera parte de este período cortado por la crisis del 2001, posiblemente los dos textos que abordaron específicamente la región de Asia Oriental, desde el aspecto económico, Dragones, Tigres y Jaguares. Relaciones América Latina/Asia Pacífico más allá de la Crisis, de Moneta y Noto, y desde el aspecto político, Argentina y el Este Asiático: La Política Exterior de 1945 a 1999, de Oviedo. Ambos textos no sólo brindaron grandes aportes en lo que hace a los conocimientos de la región de Asia Oriental en general, sino que no sólo refirieron a los países del Noreste Asiático (China, Corea, Japón) sino también a los del Sudeste Asiático, con énfasis en las vinculaciones con Argentina.

Luego de la crisis del 2001, nos encontramos con una mayor especificidad en el tratamiento académico de la región, con publicaciones dedicadas específicamente a ésta. En el año 2004 se publicó El Sudeste Asiático: Una Visión Contemporánea, por Piovani y Baglioni, luego en 2013, Argentina-ASEAN. Herramientas para una Vinculación Comercial Estratégica, por Frydman, Schuff, Báez, Massot, Battaglia y Massolo, y finalmente en 2014, Escenarios de integración, Sudeste Asiático-América del Sur. Hacia la construcción de vínculos estratégicos, por Moneta y Cesarín. También encontramos estudios ya no a nivel país sino a nivel provincial, como América Latina y el Este Asiático: Perspectivas desde Córdoba, por Santillán, Mohamed, Morero y Rubiolo, que plantean todavía una mayor especificidad por la vinculación a nivel provincial con países de Asia Oriental, incluidos los del Sudeste Asiático.

Debe considerarse también que estas producciones colectivas son en su mayoría resultado del trabajo de equipos y, en algunos casos, centros de investigación, que emergieron principalmente en la última década. Uno de los centros de investigación dedicados a los estudios del Sudeste de Asia es el CESEA (Centro de Estudio del Sudeste Asiático), que fue reinaugurado en junio de 2012 en el Instituto de Relaciones Internacionales de la Universidad Nacional de La Plata. El CESEA concentra sus actividades en la organización de seminarios, cursos y eventos académicos vinculados específicamente con los países que 
conforman la región del Sudeste de Asia. Las profusas actividades del centro son evidencia del creciente interés académico por los desarrollos económicos, políticos y culturales del SEA.

Asimismo, se han consolidado equipos de investigación en diferentes unidades académicas que abordan las relaciones de Argentina y América Latina con el Sudeste de Asia. Entre ellos encontramos el proyecto de investigación que funciona en la Universidad Siglo 21, Córdoba, desde 2012: "Política exterior y vinculaciones comerciales de Argentina con el Sudeste de Asia, China e India". También dentro del ámbito científico nacional se ha consolidado un equipo de investigación sobre las relaciones de América del Sur con el Sudeste de Asia e India, que funciona en el Centro de Investigaciones y Estudios sobre la Cultura y la Sociedad (CIECS), CONICET, dentro del Departamento de Estudios Internacionales desde 2013. Entre las producciones más destacadas de este último se encuentran artículos académicos en revistas nacionales e internacionales. Las producciones destacadas son, entre otras, "El Sudeste de Asia en las vinculaciones SurSur de la Argentina: ¿es la diversificación de socios suficiente?", en Revista de Estudos e Pesquisas sobre as Américas, Universidade de Brasilia; "Las relaciones de Argentina con Tailandia en el marco de una búsqueda de diversificación comercial", Studia Politicae, Universidad Católica de Córdoba; "Argentina-Indonesia: fortalecimiento de las relaciones comerciales y despertar del interés político en el marco de una estrategia Sur-Sur", en AD-minister, Universidad EAFIT, Medellín, Colombia.

En paralelo con el incremento de las iniciativas locales, se produjo una creciente participación con presentación de producciones individuales y colectivas en eventos académicos de alcance nacional e internacional. Entre ellos se realizaron presentaciones que son resultados parciales de las investigaciones sobre el Sudeste de Asia, en congresos de la Asociación Internacional de Ciencia Política ( IPSA Chile 2010, España 2012), y en congresos de la Asociación Latinoamericana de Ciencia Política (Argentina 2010, Ecuador 2012, Colombia 2013 y Perú 2015).

\section{Reflexiones finales}

A modo de recapitulación subrayamos, como se mencionó inicialmente, que la cuestión de los estudios del Sudeste Asiático en Argentina se sumerge en la misma historia de los estudios de las Relaciones Internacionales, y asiáticos en el país. Dentro de este contexto académico, el tratamiento de las cuestiones vinculadas al Sudeste de Asia tuvo sus sesgos particulares. Con respecto al interés de los estudios internacionales, la región fue abordada como una zona caliente en el periplo de la Guerra Fría entre Estados Unidos y la Unión Soviética, recibiendo la Guerra de Vietnam particular atención, aunque aislada del resto de los acontecimiento de la región. Con respecto al interés de los estudios asiáticos, se centró más en los 
procesos de descolonización, pero desatendiendo los consecuentes procesos de construcción del estado nación. Es de destacar la gran carencia de publicaciones específicas recientes en castellano sobre la región. Dentro de esta área vale indagar en relaciones y política exterior Argentina, con especial énfasis en su participación en organismos multilaterales como el Consejo de Seguridad y la Asamblea General de las Naciones Unidas, y el Movimiento de Países No alineados.

Entrada la década del noventa y el respectivo entrecruzamiento de las cuestiones internacionales y asiáticas, la región empieza a tener mayor atención y ser tratada como un todo. Ahora bien, nuevamente compite en desventaja con la atención recibida por los estudios sobre China, Japón, Corea, e India. Recién avanzado el siglo XXI los estudios del Sudeste Asiático empiezan a tener entidad e identidad como un todo, esto en coincidencia con el mayor protagonismo de las relaciones comerciales entre Argentina con los países de la región.

Resulta indudable que la consolidación de los mercados del Sudeste de Asia como centros económicos mundiales y, en línea con ello, como creciente destino de los esfuerzos de diversificación comercial de la Argentina $-\mathrm{y}$ de América del Sur en general- vuelve imperante un tratamiento más sistematizado $\mathrm{y}$ permanente del acontecer regional y de las vinculaciones bilaterales e interregionales. Aunque incipientes pasos se han dado desde la academia local para comenzar a construir un conocimiento actualizado y que contemple la complejidad de la región, así como las relaciones políticas y comerciales, estos esfuerzos se encuentran aún en un bajo nivel de desarrollo, y concentrados en escasos centro académicos nacionales. Consideramos, en consecuencia, que uno de los principales desafíos es la consolidación de redes académicas nacionales que permitan una construcción acumulativa e interdisciplinar de conocimiento sobre la región y sobre el acontecer de los vínculos Argentina-SEA, que contribuyan a la toma de decisiones en el ámbito público y privado para la implementación de acciones que favorezcan un acercamiento con la región.

\section{Fuentes}

BIANCO, L. (1995). Asia Contemporánea. Siglo XXI Editores: México.

BRACCIO, G. (2009). Esteban Sampzon, un escultor filipino en el Río de la Plata. Eadem Utraque Europa. Revista Semestral de Historia Cultural e Intelectual, 5(8), 53-72.

BUFFA, D. (2008). Pasado y Presente en los Estudios e Investigaciones sobre África en Argentina. En Los Estudios Afroamericanos y Africanos en América Latina: Herencia, Presencia y Visiones del Otro (pp. 341- 
355). Buenos Aires: Centro de Estudios Avanzados del Consejo Latinoamericano de Ciencias Sociales. Recuperado de http://biblioteca.clacso.edu.ar/clacso/coediciones/20100823035418/19buffa.pdf

BULCOURF, P.; Cardozo, N. y Campos Ríos, M. (2013). Los Estudios Internacionales en la Argentina en Perspectiva Histórica. Revista Aportes para la Integración Latinoamericana, XIX (29), 31-64. Recuperado de http://sedici.unlp.edu.ar/bitstream/handle/10915/37212/Documento completo.pdf?sequence=1

CAILLET-BOIS, R. (1930). Nuestros Corsarios. Brown y Bouchard en el Pacífico (1815-1816). Buenos Aires: UBA.

CICHERO, D. (1999). El Corsario del Plata: Hipólito Bouchard y su Viaje alrededor del Mundo. Buenos Aires: Sudamericana.

CRESPI, L. (2010). En busca de un enclave esclavista. La expedición colonizadora a las islas de Fernando Poo y Annobon, en el Golfo de Guinea. (1778 - 1782). Revista Digital Estudios Históricos del Centro de Documentación Histórica del Río de la Plata, "Prof. Dr. Walter Rela", II (4), Recuperado de http://www.estudioshistoricos.org/edicion 4/liliana-crespi.pdf

(2001). Comercio de Esclavos en el Río de la Plata Durante el Siglo XVII. En CÁCERES, R. (Ed.). Rutas de la Esclavitud en África y América Latina (pp. 101-113). San José de Costa Rica: Editorial de la Universidad de Costa Rica.

DEVOTO, L. y PELOSI, H. (2010). Viaje del presidente Frondizi a Canadá y Asia. Temas de historia argentina y americana, 16, 147-176. Recuperado de http://bibliotecadigital.uca.edu.ar/repositorio/revistas/viajefrondizi-canada-asia.pdf

DI MASI, J. (1999). Informe sobre la participación de la Universidad Nacional de La Plata en la Primera Comisión Mixta Argentina-Vietnam y en la Misión a la República Popular Democrática de Laos y a la Unión de Myanmar. Relaciones Internacionales del Instituto de Relaciones Internacionales, 17. Recuperado de http://sedici.unlp.edu.ar/bitstream/handle/10915/9960/Documento completo.pdf?sequence=1

Dirección Nacional de Población (2012). Refugiados del Sudeste Asiático en la Argentina: 30 Años de Historia. En Registro Nacional de las Personas, Ministerio del Interior y Transporte. Recuperado de http://www.mininterior.gov.ar/poblacion/pdf/Documento07.pdf

Dirección Nacional de Solidaridad Argentina con Vietnam (1971). 7 Puntos para la paz en Indochina. Buenos Aires: Dirección Nacional de Solidaridad Argentina con Vietnam.

(1970). Frente al enemigo común: unidos en un mismo deseo de paz e independencia nacional. Buenos Aires: Dirección Nacional de Solidaridad Argentina con Vietnam.

(1969). Sí, Hay un Camino para la Paz en Vietnam. Buenos Aires: Dirección Nacional de Solidaridad Argentina con Vietnam. 
(1968). Los Argentinos ante el Drama de Vietnam. Buenos Aires: Dirección Nacional de Solidaridad Argentina con Vietnam.

(1966). Testimonio sobre Vietnam. Buenos Aires: Dirección Nacional de Solidaridad Argentina con Vietnam.

El Tiempo (1961). Viaje alrededor del mundo inicia hoy Arturo Frondizi (27 de noviembre). Recuperado de http://news.google.com/newspapers

$\underline{\text { ?nid }=1706 \& \text { dat }=19611127 \& i d=s c A q A A A A I B A J \& s j i d=82 g E A A A A I B A J \& p g=4786,652488}$

Escuela de Estudios Orientales (2015). Reseña de la Facultad. Disponible en el sitio web de la Universidad del Salvador, http://eeo.usal.edu.ar/eeo/resena-de-la-facultad

FRYDMAN,F; SCHUFF, M; BÁEZ, G; MASSOT, J. M.; BATTAGLIA, M. Y MASSOLO, M. (2013). ArgentinaASEAN. Herramientas para una Vinculación Comercial Estratégica. Buenos Aires: CARI.

MARILUZ URQUIJO, J. M. (1984). La China, Utopía Rioplatense del Siglo XVIII. Revista de Historia de América del Instituto Panamericano de Geografía e Historia, 98, 7-31. Recuperado de http://www.larramendi.es/i18n/catalogo imagenes/grupo.cmd?path=1000243

MEDINA, J. T. (1928). La Expedición de Corso del Comodoro Guillermo Brown : En Aguas del Pacífico (Octubre de 1815-Junio de 1816). Buenos Aires: Peuser.

MONETA, C. Y CESARÍN, S. (2014). Escenarios de integración, Sudeste Asiático-América del Sur. Hacia la construcción de vínculos estratégicos. Buenos Aires: Eduntref.

MONETA, C. Y NOTO, G. O. (1998). Dragones, Tigres y Jaguares. Relaciones América Latina/Asia Pacífico más allá de la Crisis. Buenos Aires: Corregidor.

MREC (1984). Convenio comercial entre el gobierno de la República Argentina y el gobierno de la República de Filipinas. Disponible en el Subsitio de la Biblioteca Digital de Tratados del Sitio del Ministerio de Relaciones Exteriores y Culto de la República Argentina, http://tratados.mrecic.gov.ar/busqueda.php?consulta=si\&modo=c

MREC (2015). Subsitio de la Biblioteca Digital de Tratados del Sitio del Ministerio de Relaciones Exteriores y Culto de la República Argentina. Disponible en:

http://tratados.mrecic.gov.ar/busqueda.php?consulta $=$ si\&modo=c

O’ DONNELL, P. (2006). Historias Argentinas: de la Conquista al Proceso. Buenos Aires: Sudamericana.

(2004). Los Héroes Malditos: La Historia Argentina que no nos Contaron. Buenos Aires:

Sudamericana.

OVIEDO, E.D. (2001). Argentina y el Este Asiático. La Política Exterior Argentina de 1945 a 1999. Rosario: UNR Editora. 
(2000). La política exterior argentina hacia el Asia oriental 1983-1999: consideraciones y aspectos principales. DTI, 3. Buenos Aires: Consejo Argentino para Las Relaciones Internacionales. Recuperado de http://www.cari.org.ar/publicaciones/pdf/ argentina-asia.pdf PANNIKAR, K. M. (1966). Asia y la Dominación Occidental. Un Examen de la Historia de Asia desde la Llegada de Vasco da Gama (1498.1945). Buenos Aires: Editorial Universitaria de Buenos Aires.

PAZ, G. (2005). Argentina's Relations with East Asia. En Faust, J., Mols, M.y Won-Ho, K.(Eds.). Latin America and East Asia - Attemps at Diversification (pp. 45-64). Seul: Korea Institute for International Economic Policy.

PELOSI, H. C. Y MACHINANDIARENA DE DEVOTO, L.. (2012). Las relaciones internacionales en la presidencia de Frondizi : desarrollo, integración latinoamericana y paz mundial. Saarbrücken : Lap Lambert Academic PublishingEditorial Académica Española. Recuperado de

http://bibliotecadigital.uca.edu.ar/repositorio/libros/relacionesinternacionales-presidencia-frondizi.pdf

PIOVANI, J. I. Y BAGLIONI, S. D. (2004). El Sudeste Asiático: Una Visión Contemporánea. Buenos Aires: Eduntref.

POLVERINI, A. (1968). La danza de las noctilucas : La fragata Libertad por mares y puertos del mundo. Buenos Aires: Centro Naval.

SANTILLÁN, G; MOHAMED, N; MORERO, H. Y RUBIOLO, F (Eds.). (2011). América Latina y el Este Asiático: Perspectivas desde Córdoba. Córdoba: CIECS-CONICET.

QUARTARUOLO, M. (1967). Fragata La Argentina: su vuelta al mundo (1817-1819). Buenos Aires: Departamento de Estudios Históricos Navales.

SAAVEDRA, M. (2004). La Argentina no Alineada: desde la Tercera Posición Justicialista hasta el Menemismo, 1973-1991. Buenos Aires: Biblos.

SIMONOFF, A. (2010). La política exterior de Arturo Illia: el krausismo renovado (1963-1966). En Simonoff, A. (Comp.). La Argentina y el mundo frente al bicentenario de la Revolución de mayo (pp. 233-257). La Plata: EDULP.

(2008). La Política Exterior de los Gobiernos Kirchneristas y la Tercera Posición. Revista Intellector del Centro de Estudios en Geopolítica y Relaciones Internacionales, IV, V (9), 1-20.

UBA (2015). Subsitio del Grupo de Estudios del Este Asiático. Instituto de Investigaciones Gino Germani. Disponible en http://webiigg.sociales.uba.ar/geea/qsomos1.htm

Universidad Nacional de Tres de Febrero (2015). Subsitio del CEAPI. Disponible en http://untref.edu.ar/institutos centros/ceapi-centro-de-estudios-sobre-asia-del-pacifico-e-india/ 
US Department of State (2012). Office of the Historian. Disponible en http://history.state.gov/departmenthistory/visits/argentina 